\title{
An international registry for primary ciliary dyskinesia
}

\author{
Claudius Werner ${ }^{1}$, Martin Lablans ${ }^{2}$, Maximilian Ataian ${ }^{2}$, Johanna Raidt ${ }^{1}$, \\ Julia Wallmeier ${ }^{1}$, Jörg Große-Onnebrink ${ }^{1}$, Claudia E. Kuehni ${ }^{3}$, \\ Eric G. Haarman ${ }^{4}$, Margaret W. Leigh ${ }^{5}$, Alexandra L. Quittner ${ }^{6}$, Jane S. Lucas ${ }^{7}$, \\ Claire Hogg ${ }^{8}$, Michal Witt ${ }^{9}$, Kostas N. Priftis ${ }^{10}$, Panayiotis Yiallouros ${ }^{11}$, \\ Kim G. Nielsen ${ }^{12}$, Francesca Santamaria ${ }^{13}$, Frank Ückert ${ }^{2}$ and Heymut Omran ${ }^{1}$
}

Affiliations: 'Dept of General Paediatrics, Paediatric Pulmonology Unit, University Children's Hospital Muenster, Münster, Germany. ${ }^{2}$ Institute of Medical Biostatistics, Epidemiology and Informatics, University Medical Center of Johannes Gutenberg University Mainz, Mainz, Germany. ${ }^{3}$ Institute of Social and Preventive Medicine (ISPM), Paediatric Respiratory Epidemiology, University of Bern, Bern, Switzerland. ${ }^{4}$ Dept of Paediatric Pulmonology, VU University Medical Center, Amsterdam, The Netherlands. ${ }^{5}$ Dept of Pediatrics, University of North Carolina at Chapel Hill School of Medicine, Chapel Hill, NC, USA. ${ }^{6}$ Dept of Psychology, University of Miami, Coral Gables, FL, USA. ${ }^{7}$ University of Southampton Faculty of Medicine and University Hospital Southampton NHS Foundation Trust, Southampton, UK. ${ }^{8}$ Dept of Respiratory Paediatrics, Royal Brompton Hospital, London, UK. ${ }^{9}$ Dept of Molecular and Clinical Genetics, Institute of Human Genetics, Polish Academy of Sciences, Poznan and International Institute of Molecular and Cell Biology, Warsaw, Poland. ${ }^{10}$ Paediatric Pulmonology, 3rd Dept of Paediatrics, National and Kapodistrian University of Athens, School of Medicine, Attikon University Hospital, Athens, Greece. ${ }^{11}$ Cyprus International Institute for Environmental \& Public Health in Association with Harvard School of Public Health, Cyprus University of Technology, Limassol, Cyprus. ${ }^{12}$ Danish PCD Centre, Paediatric Pulmonary Service, Dept of Paediatrics and Adolescent Medicine, Copenhagen University Hospital, Copenhagen, Denmark. ${ }^{13}$ Dept of Medical Translational Sciences, Federico II University, Naples, Italy.

Correspondence: Claudius Werner, Dept of General Paediatrics, University Children's Hospital Muenster, Albert-Schweitzer-Campus 1, Geb. A1, 48149 Münster, Germany. E-mail: claudius.wernerQukmuenster.de

ABSTRACT Primary ciliary dyskinesia (PCD) is a rare autosomal recessive disorder leading to chronic upper and lower airway disease. Fundamental data on epidemiology, clinical presentation, course and treatment strategies are lacking in PCD. We have established an international PCD registry to realise an unmet need for an international platform to systematically collect data on incidence, clinical presentation, treatment and disease course.

The registry was launched in January 2014. We used internet technology to ensure easy online access using a web browser under www.pcdregistry.eu. Data from 201 patients have been collected so far. The database is comprised of a basic data form including demographic and diagnostic information, and visit forms designed to monitor the disease course.

To establish a definite PCD diagnosis, we used strict diagnostic criteria, which required two to three diagnostic methods in addition to classical clinical symptoms. Preliminary analysis of lung function data demonstrated a mean annual decline of percentage predicted forced expiratory volume in $1 \mathrm{~s}$ of $0.59 \%$ (95\% CI 0.98-0.22).

Here, we present the development of an international PCD registry as a new promising tool to advance the understanding of this rare disorder, to recruit candidates for research studies and ultimately to improve PCD care.

@ERSpublications

A registry to systematically collect data on clinical presentation, disease course and treatment of PCD http://ow.ly/TtGAR

A press release for this article is available from erj.ersjournals.com/press

Received: May 182015 | Accepted after revision: Oct 072015 | First published online: Dec 092015

Clinical trial: This study is registered at www.clinicaltrials.gov with identifier number NCT02419365.

Support statement: This work was supported by European Commission FP7 grant no. 305404 (BESTCILIA). Funding information for this article has been deposited with FundRef.

Conflict of interest: Disclosures can be found alongside the online version of this article at erj.ersjournals.com

Copyright CERS 2016 


\section{Introduction}

Primary ciliary dyskinesia (PCD (Online Mendelian Inheritance in Man: 244400)) is a rare autosomal recessive disorder of ciliary motility resulting in chronic upper- and lower-airway disease. Organ laterality defects occur in $\sim 40-50 \%$ of cases, usually situs inversus totalis (the condition is then known as Kartagener's syndrome). PCD affects $\sim 1: 20000$ individuals with a reported prevalence of 1:4000 to $<1: 50$ 000 [1]. PCD is genetically heterogeneous. So far, mutations in 31 different genes have been identified to be causative for this disorder [2-6] accounting for $\sim 60-70 \%$ of cases. Due to biological and phenotypical heterogeneities, different diagnostic tests have to be combined to confirm a PCD diagnosis [5-7]. As access to diagnostic facilities is scarce in many countries, PCD is under-diagnosed and diagnosed too late [1].

While tremendous progress has been made in recent years in unveiling the molecular mechanisms of the disease [2, 3, 8-19], PCD management is still hampered by a lack of fundamental data on epidemiology, clinical presentation, disease course and effects of different treatment strategies [4-6]. Patient registries are well-known tools to assemble sufficient numbers of individuals with a rare disease to assess and monitor patient data in a standardised and longitudinal way, and to recruit candidates for clinical research studies $[20,21]$. Patient data have been collected by PCD centres on national or regional levels, e.g. in Denmark, UK, Switzerland, USA and Germany. These databases have proved indispensable in facilitating research aimed at the identification of novel PCD variants. By contrast, epidemiological and clinical research requires larger datasets of items collected in a highly standardised way. In order to better characterise the clinical course, and improve the diagnosis and treatment of PCD patients, we have established an internet-based, international PCD registry for systematic and longitudinal data collection. The goal of the registry is to collect data on a large number of individuals to advance our understanding of the range of conditions associated with PCD and the factors that contribute to health outcomes. The objectives of the present article are to: 1) detail the scientific rationale used to implement the registry, 2) describe the methodological details of registry design, patient recruitment, data collection and data management, 3) raise awareness of this novel tool, and 4) report first data on lung function and methods used for establishing a PCD diagnosis.

\section{Methods}

Rationale and requirements for the PCD registry

The international PCD registry was established to realise a previously unmet research need as there was no international platform to systematically collect data from PCD individuals on the incidence, clinical presentation, treatment and course of the disease. Importantly, the registry does not only serve clinicians and researchers, but also provides the opportunity for individuals to participate in research who have been under-represented previously.

The aims of the PCD registry include specifically the collection of data about diagnostic certainty, health status at time of diagnosis, progression of disease (including influence of specific management plans), severe infections, colonisation with problem microbes, life expectancy, sex-specific differences (e.g. fertility), subtypes with unique clinical course as well as quality of life.

To achieve high acceptance of the registry, the registry was designed in close collaboration with lead clinicians and researchers in Europe and North America as well as European and North American PCD support groups. As legal, data protection and ethical requirements vary widely within different countries, electronic data collection is restricted to anonymised data.

\section{Eligibility criteria and recruitment}

The PCD patient registry is a longitudinal study. It recruits all prevalent diagnosed subjects with PCD and all subsequent incident cases. Both children and adults with PCD are eligible. Patients who fulfil the following diagnostic criteria are considered to have a definite PCD diagnosis: 1) clinical presentation consistent with PCD and 2) consistent findings specific for PCD by at least two methods (high-frequency video microscopy analysis (HVMA), transmission electron microscopy (TEM), immunofluorescence microscopy (IF), low nasal nitric oxide concentration/production (nNO) or bi-allelic disease-causing mutations by genotyping).

If only HVMA and nNO are abnormal, HVMA should be repeated at least three times and show the same abnormal results each time. Given the complexity of diagnosing PCD, it is anticipated that not all patients will meet these definite diagnostic criteria. Therefore, all individuals with typical clinical symptoms are eligible to enter the registry. These cases are usually considered to have a probable (one abnormal diagnostic test) or possible (no clearly abnormal test) PCD diagnosis with exceptions made on an individual basis (e.g. identification of the same bi-allelic disease-causing mutations in a subject of a sibling with a confirmed diagnosis). 
Patients are recruited by their medical care-givers who are assigned as a referring centre. Becoming a referring centre involves meeting all necessary legal and ethical requirements, a process guided by the registry management team. Entering individual patient data is possible after written informed consent has been obtained by the patient or his/her legal guardians. Informed consent forms are kept by the referring centre to ensure complete anonymity of data collected in the electronic registry. No information is retained from participants who do not consent. Participants can withdraw their authorisation for participation in the study at any time.

All protocols for data collection and informed consent have been approved by the Ethical Review Committees of the Medical Council Westfalen-Lippe and the Medical Faculty of the Westphalian Wilhelms University Münster.

\section{Item generation, programming of the registry database and data protection}

Agreement on the items collected in the registry has been achieved by integrating input from representatives of the US and several European PCD support groups. An internet-based data entry system has been developed that provides plausibility checks. Furthermore, the system has been prepared to integrate data from already existing local databases using suitable computer interfaces, thus avoiding the need to enter similar data in two different databases. The database is securely located at the Institute of Medical Biostatistics, Epidemiology and Informatics, Mainz (IMBEI), University Medical Centre Mainz, Germany. This institute is responsible for maintenance, technical support and data protection of the registry. Backup of data takes place daily using multiple servers. The registry is protected against misuse by various security measures, such as firewall protection, encrypted data transfer and password-protected access to the database. Except for the administration team, each contributor only has direct access to his/ her own data. A master list containing information that links the subject ID to identifying patient data is not collected in the PCD registry. Such a list is kept peripherally by the referring centres.

\section{Quality assurance}

To ensure a high level of data quality, a three-step quality assurance process is employed that complements plausibility checks provided by the registry software with two steps of manual control. 1) Data entry performed by each centre's operators is supported by the registry's automatic validation routines. 2) All nine forms that constitute a questionnaire are reviewed and explicitly finalised ("signed") by the operator. 3) These finalised forms are reviewed again by the registry administrator team. Only forms that have passed all three checks are considered valid and included in subsequent evaluations.

\section{Data utilisation and sharing plan}

Annual registry reports will be prepared using standard statistical software. A detailed description of all relevant outcome parameters will be given applying descriptive statistical methods, i.e. frequency tables, location and scale statistics and graphical presentations. Investigators interested in accessing registry data or in recruiting registry participants for research studies are invited to contact the registry's steering committee. Short concept proposals can be submitted which will be discussed for feasibility and scientific impact by the registry steering committee. Importantly, utilisation of patient data always requires explicit written approval by the referring centres who have provided data and, if not covered by the signed informed consent for the registry, by the affected PCD subjects.

\section{Statistical analysis}

SPSS 22 (www.ibm.com) and Prism 6 (www.graphpad.com) were employed to calculate all statistics and to summarise the demographic characteristics of participants.

\section{Results}

\section{Registry items}

The database is comprised of a basic data form (table 1) including demographic and diagnostic information, and seven visit forms (table 2) covering items to monitor the disease course. While basic data are changed only when applicable, visit data are updated regularly (visit data can be entered at every visit; yearly updates are requested). A diagram of the registry design is given in figure 1 .

Registry items include age at diagnosis, family history (consanguinity), associated malformations/diseases, laterality defects, clinical manifestations (otitis media, rhinosinusitis, pneumonia, bronchiectasis, neonatal respiratory distress syndrome), microbiological results, diagnostic findings (HVMA, TEM, nNO, IF, genotype), lung function, radiological findings and therapeutic measures (inhalation, antibiotics, oxygen, ventilation, ENT surgery, lung surgery). Furthermore, quality-of-life assessment using QOL-PCD, i.e. age-appropriate PCD-specific patient-reported outcome questionnaires developed in another BESTCILIA 
TABLE 1 Basic registry data: items collected at baseline; new information is added when appropriate

Items

Administrative data

Family history

Symptoms leading to diagnosis

Male fertility

Female fertility

Primary ciliary dyskinesia diagnostics
Written informed consent

Patient identification; centre identification

Birth month, birth year

Gender

Ethnic origin

Socioeconomic background: level of education (parents and patient)

Parental consanguinity

Affected siblings

Other affected family members

History of neonatal respiratory distress syndrome

Situs inversus totalis

Chronic rhinitis/rhinosinusitis

Chronic/recurrent otitis media

Chronic wet cough

Chronic/recurrent lower airways infection

Chronic atelectasis

Heterotaxia

Congenital heart defect

Cystic kidney disease

Hearing loss

Male infertility

Hydrocephalus internus

Retinitis pigmentosa

Number of children, semen analysis, use of assisted reproductive technologies

Number of pregnancies, number of miscarriages, number of ectopic pregnancies, number of children, use of assisted reproductive technologies

Age, length/height and weight at diagnosis

Nasal nitric oxide measurement

High-frequency video microscopy findings

Transmission electron microscopy analysis

High-resolution immunofluorescence findings

Genetic results

All items contain the input option: not done, not available or not relevant.

work package (www.bestcilia.eu), will be incorporated in the database as soon as validation in different languages is completed.

\section{Enrolment and demographic characteristics}

The registry was launched in January 2014 (www.clinicaltrials.gov: NCT02419365). It is accessible via a web browser under www.pcdregistry.eu. So far, data from 201 patients from five referring centres have been collected in the database. Recruitment rates increased gradually from one patient in the first month to a maximum of 46 patients in January 2015 (figure 2). As it is also possible to enter retrospective data, data for more than one visit have been provided for 36/201 individuals. Gender distribution was 110 (54.7\%) females and 91 (45.3\%) males. Out of the 201 individuals, 98 (48.8\%) were under 18 years old and 103 (51.3\%) were adults. The median age of participants was 18 years with a range from 1 month to 76 years. The age distribution is shown in figure 3.

\section{Establishment of PCD diagnosis}

Individuals were classified as having a definite, probable or possible PCD diagnosis as described in the eligibility criteria in the Methods section. Out of 201 individuals, 123 had a definite diagnosis, 33 were graded as subjects with a probable PCD diagnosis and 45 were assigned to have a possible PCD diagnosis (figure 4).

Diagnostic tests used for PCD work-up included nNO, HVMA, TEM, IF and genetic analysis (figure 5). HVMA was most commonly used (144 individuals, 70\%) and was abnormal in 140 individuals. HVMA 
TABLE 2 Visit data items collected at least yearly

Field Items

Anthropometric data

Lung function

Imaging

Clinical disease manifestation

\section{Microbiological findings}

Therapy
Items

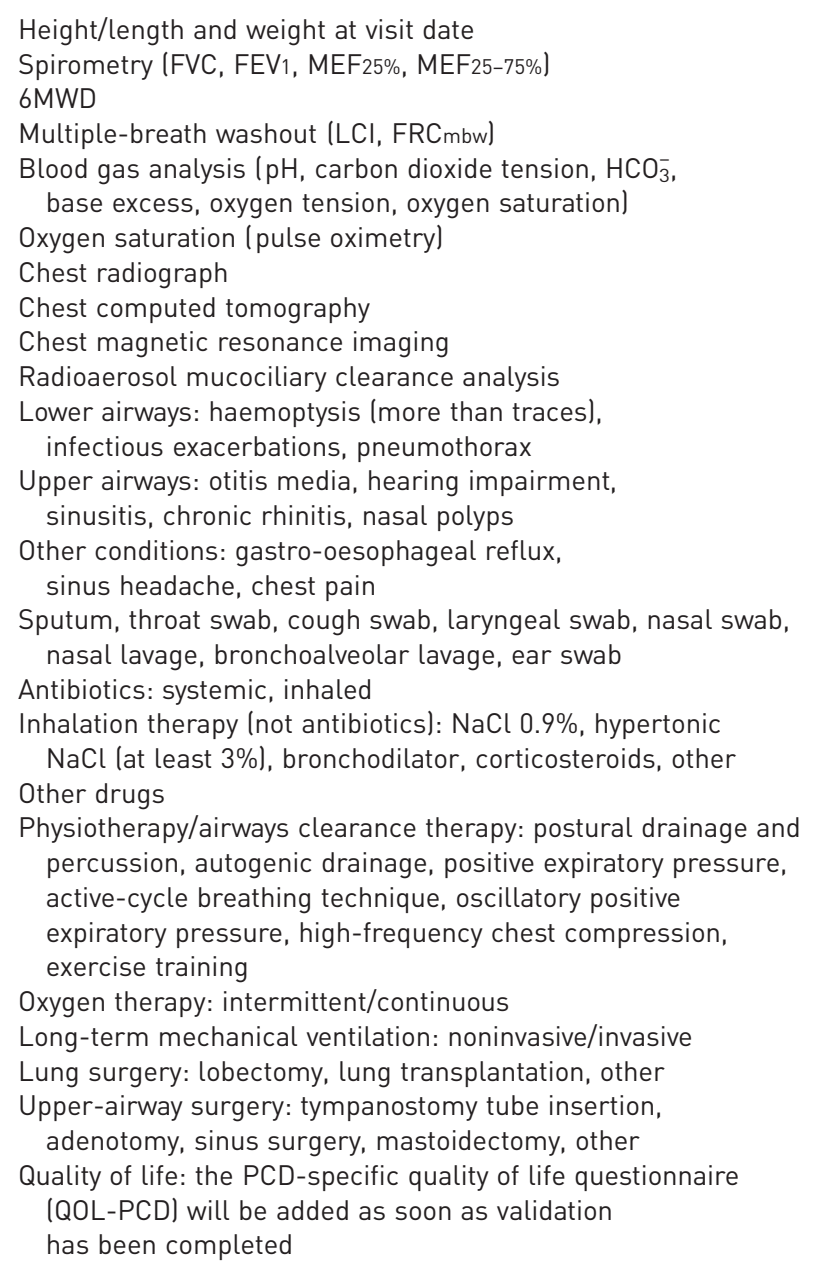

All items contain the input option: not done, not available or not relevant. Users can choose between this extended dataset and a limited dataset with fewer variables. FVC: forced vital capacity; FEV1: forced expiratory volume in $1 \mathrm{~s}$; MEF25\%: mid-expiratory flow at $25 \%$ of FVC; MEF25-75\%: mid-expiratory flow at $25-75 \%$ of FVC; 6MWD: 6-min walking distance; LCl: lung clearance index; FRC: functional residual capacity; PCD: primary ciliary dyskinesia.

was scored normal only in four individuals (2\%). All these four individuals fell into the category of possible PCD diagnosis. Among the 142 subjects with nNO testing, 10 individuals (5\%) exhibited normal results. Three of these subjects with normal nNO values were rated as having a definitive PCD diagnosis based on other tests. TEM showed normal results in 30 out of 119 tested individuals (25.2\%). IF was performed in 69 individuals. In 48 patients, IF showed clearly abnormal findings, whereas IF staining was normal in 21 individuals. Of note, the number of antibodies, which correlates with the sensitivity of the method, used for IF varied from two to six. Genetic results were available in a subset of 40 individuals, 30 of which displayed bi-allelic disease-causing mutations.

The number of diagnostic tests used in each patient is given in figure $5 f$. The majority of individuals had two $(n=50 ; 25 \%)$ or three $(n=61 ; 30 \%)$ tests.

\section{Lung function in PCD}

In a subset of 71 individuals with definite PCD, lung function data have already been cleared (figure 6). Linear regression of percentage predicted forced expiratory volume in $1 \mathrm{~s}$ (FEV1 \% pred) values versus age exhibited a mean annual decline of $0.59 \%$ ( $\mathrm{p}=0.0025$; 95\% CI $0.98-0.22$ ). Mean percentage predicted forced vital capacity (FVC \% pred) decline was $0.39 \%$ per year, which was not statistically significantly 
different from zero ( $\mathrm{p}=0.0851 ; 95 \%$ CI $0.83-0.06$ ). Compared with published data [22], our cohort showed a similar decline of FEV1 \% pred. As our FEV1 \% pred data did not differ significantly from MARTHIN et al. [22] (Mann-Whitney test, $\mathrm{p}=0.3570$ ), we pooled data from both studies and calculated a yearly decline of FEV1 \% pred of $0.72 \%$ in this cohort of 142 individuals $(\mathrm{p}<0.0001$; 95\% CI 0.98-0.46).

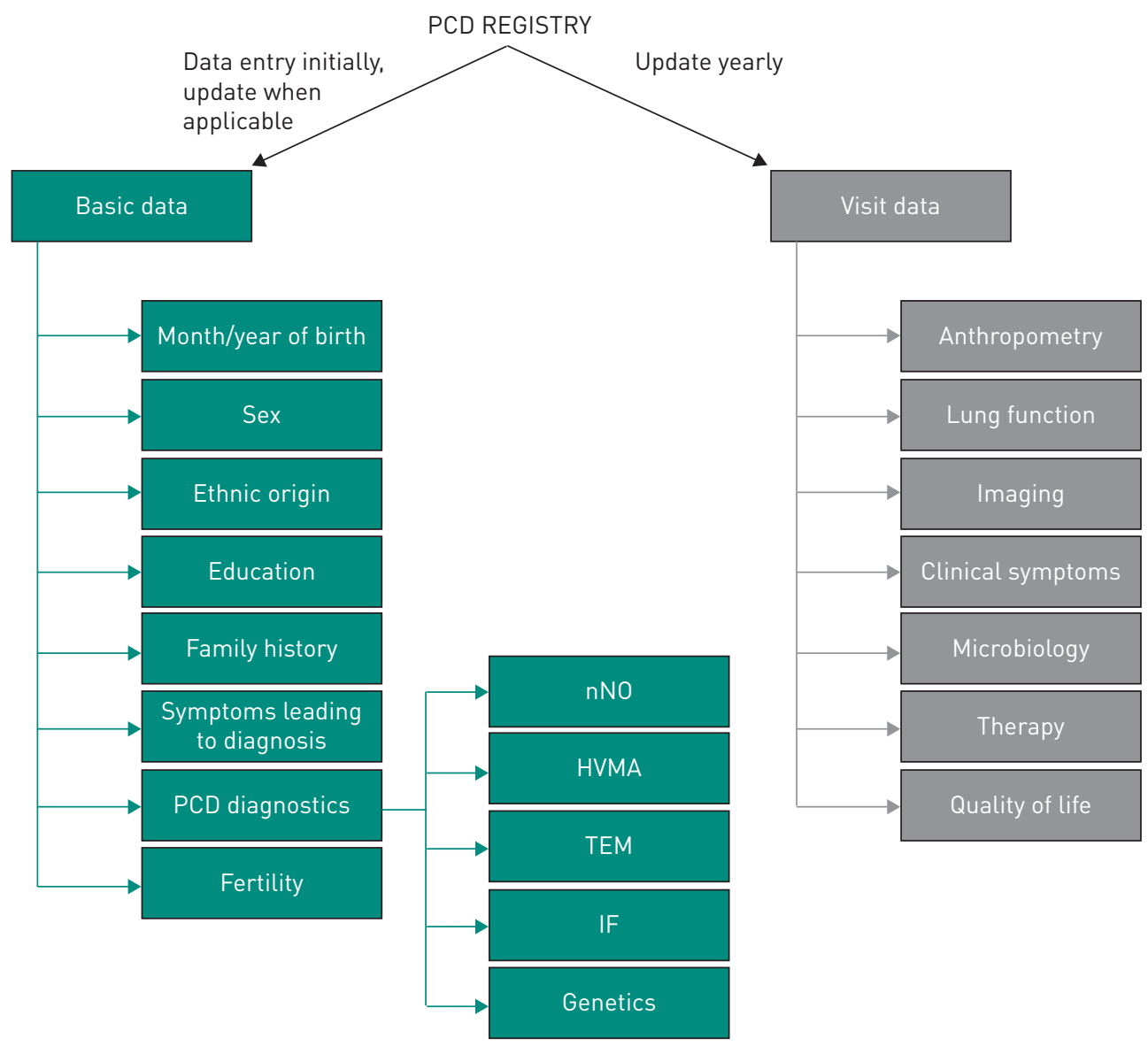

FIGURE 1 Primary ciliary dyskinesia (PCD) registry design. The database is comprised of a basic data form that is completed at recruitment and changed only when applicable. Visit data are updated at least once a year. nNO: nasal nitric oxide production rate/concentration; HVMA: high-frequency video microscopy analysis; TEM: transmission electron microscopy; IF: immunofluorescence microscopy.

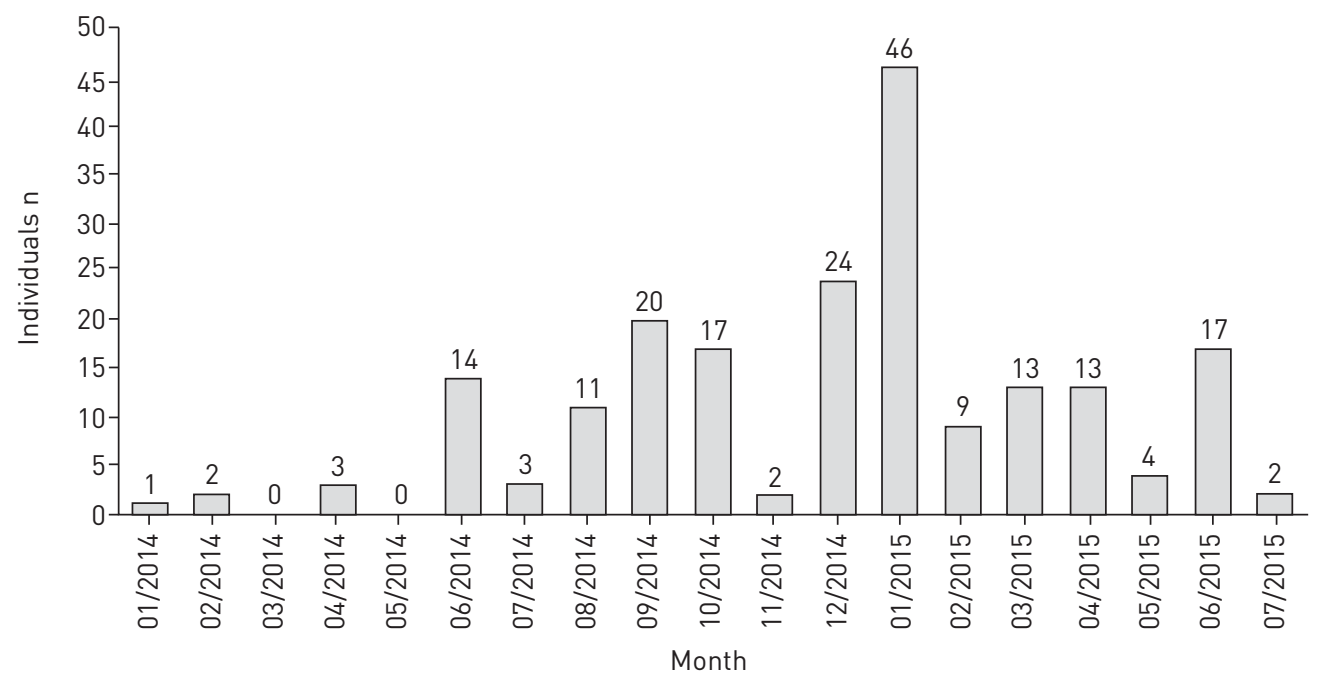

FIGURE 2 Recruitment of patients per month from January 2014 to July 2015. Total participants n=201. 
FIGURE 3 Age distribution of individuals. Total participants $\mathrm{n}=201$.

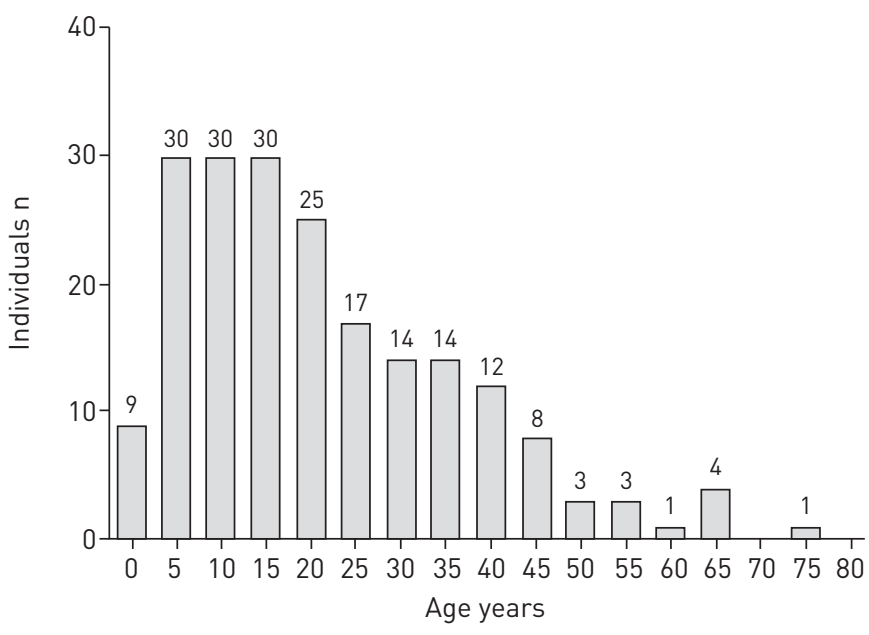

\section{Discussion}

PCD is a rare congenital respiratory disorder presenting to clinicians and patients with numerous uncertainties. As robust data describing disease severity and course are largely lacking, management has to rely on small retrospective, cross-sectional case series and experiences in disorders such as cystic fibrosis or non-cystic fibrosis bronchiectasis. This approach, however, is unsafe, because it neglects differences in the pathophysiology of these diseases. Therefore, we have established an international PCD registry to address the urgent need to systematically assess epidemiological, clinical and psychological parameters associated with PCD and its management in a sufficient number of individuals. This will not only give a detailed picture of the current disease status, but will also identify effects of changes in PCD diagnostics and treatment. Furthermore, the registry will facilitate the interconnection of PCD centres, and will serve as a tool to identify and recruit suitable individuals for research studies.

A main strength of the registry is its collaborative development. Selection of registry items has not only been agreed on among participating centres, but has also been harmonised with patient organisations, including the North American PCD Foundation. Therefore, it is guaranteed that information collected in the registry will be highly relevant and that the patient's perspective is appropriately represented.

In order to achieve the highest possible acceptance, we took particular care to create a user-friendly environment. Data entry is possible via a standard web browser. Thus, there is no need to install additional software. As part of the data security plan, access to the database is provided via user-specific accounts that allow tracking back any change in the database to the responsible person. Users of a given centre only have access to patient data of their own centre.

A unique feature of the registry is the assessment of quality of life as an important patient reported outcome measure. Currently, PCD-specific quality-of-life questionnaires (QOL-PCD) in different languages

FIGURE 4 Level of diagnostic certainty was definite in 123 (61\%), probable in $33(17 \%)$ and possible in $45(22 \%)$ individuals.

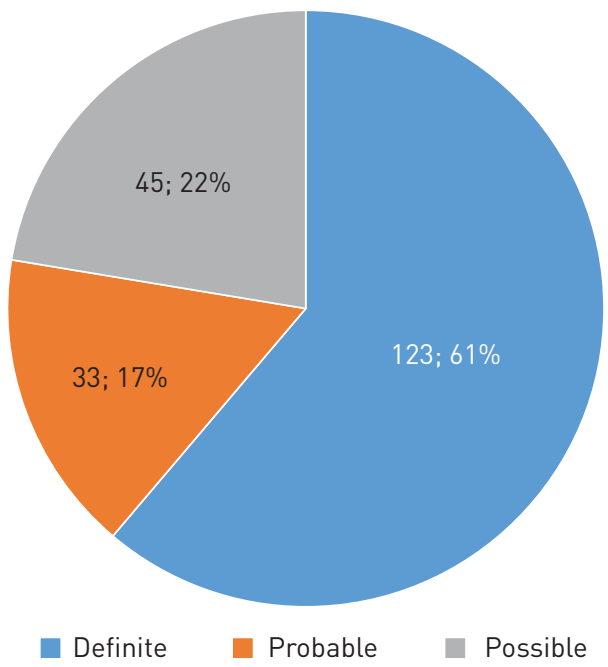


a)

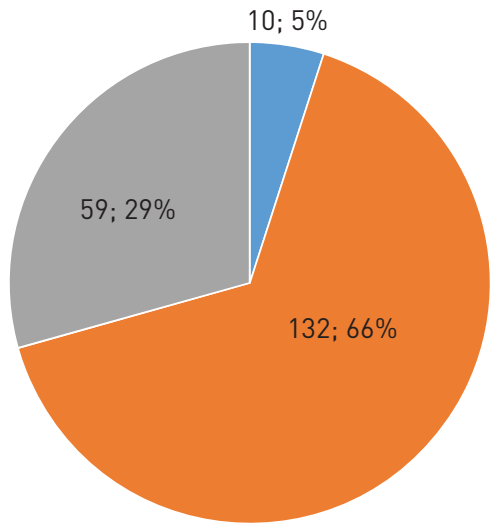

Normal

Abnormal

c)

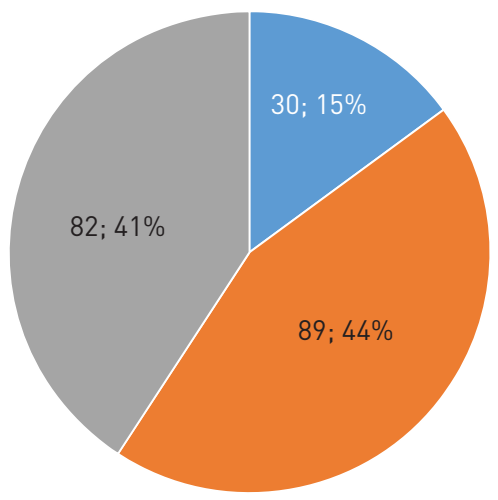

Normal

Abnormal

$10 ; 5 \%$

e)

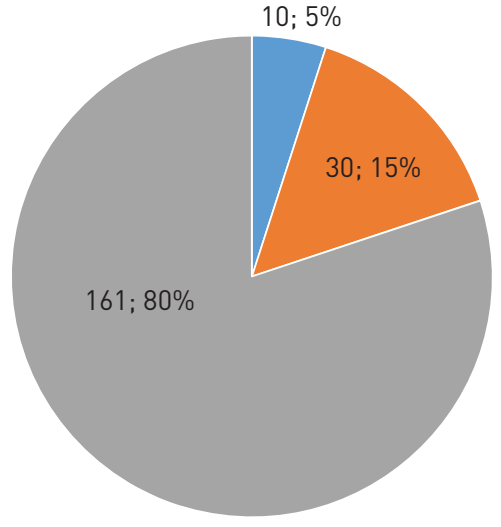

Normal Abnormal
Not available

Not available b)

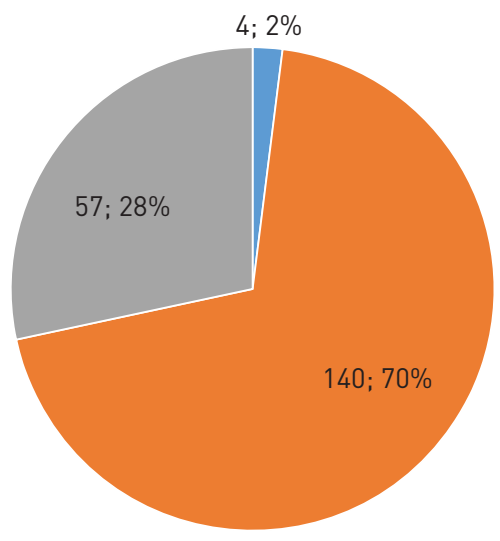

Normal Abnormal Not available

d)

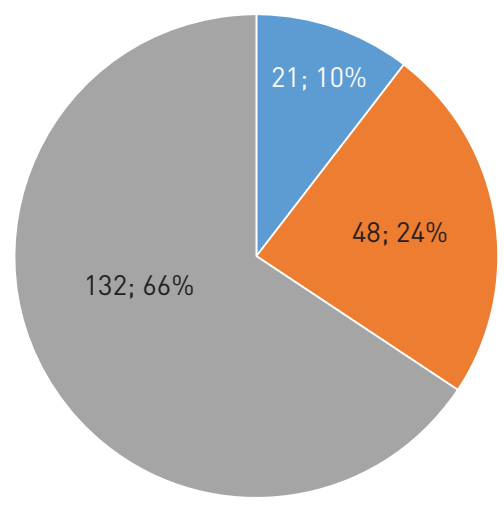

Normal Abnormal Not available

f)

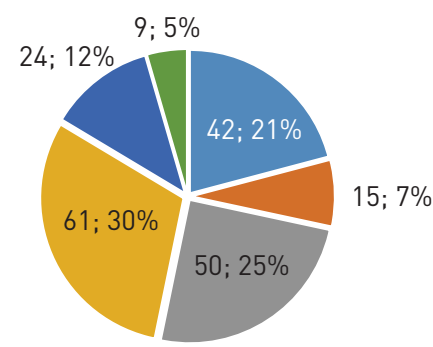
No abnormal test
- 1 abnormal test
2 abnormal tests
3 abnormal tests
4 abnormal tests
5 abnormal tests

FIGURE 5 Number and percentage of normal and abnormal test results using different methods. a) Nasal nitric oxide production rate/concentration. b) High-frequency video microscopy analysis. c) Transmission electron microscopy. d) Immunofluorescence microscopy. e) Genetic identification of bi-allelic disease-causing mutations. f) Number and percentage of individuals with zero, one, two, three four and five abnormal test results. Results from all five tests were available only in a subset of individuals. In most subjects, findings from two to three tests were available.

are being validated [23]. As soon as this process is completed in the different languages represented in the registry, these questionnaires will be incorporated in the registry. Therefore, we will include a sensitive tool to measure symptoms, functioning and quality of life on a routine basis [24]. 

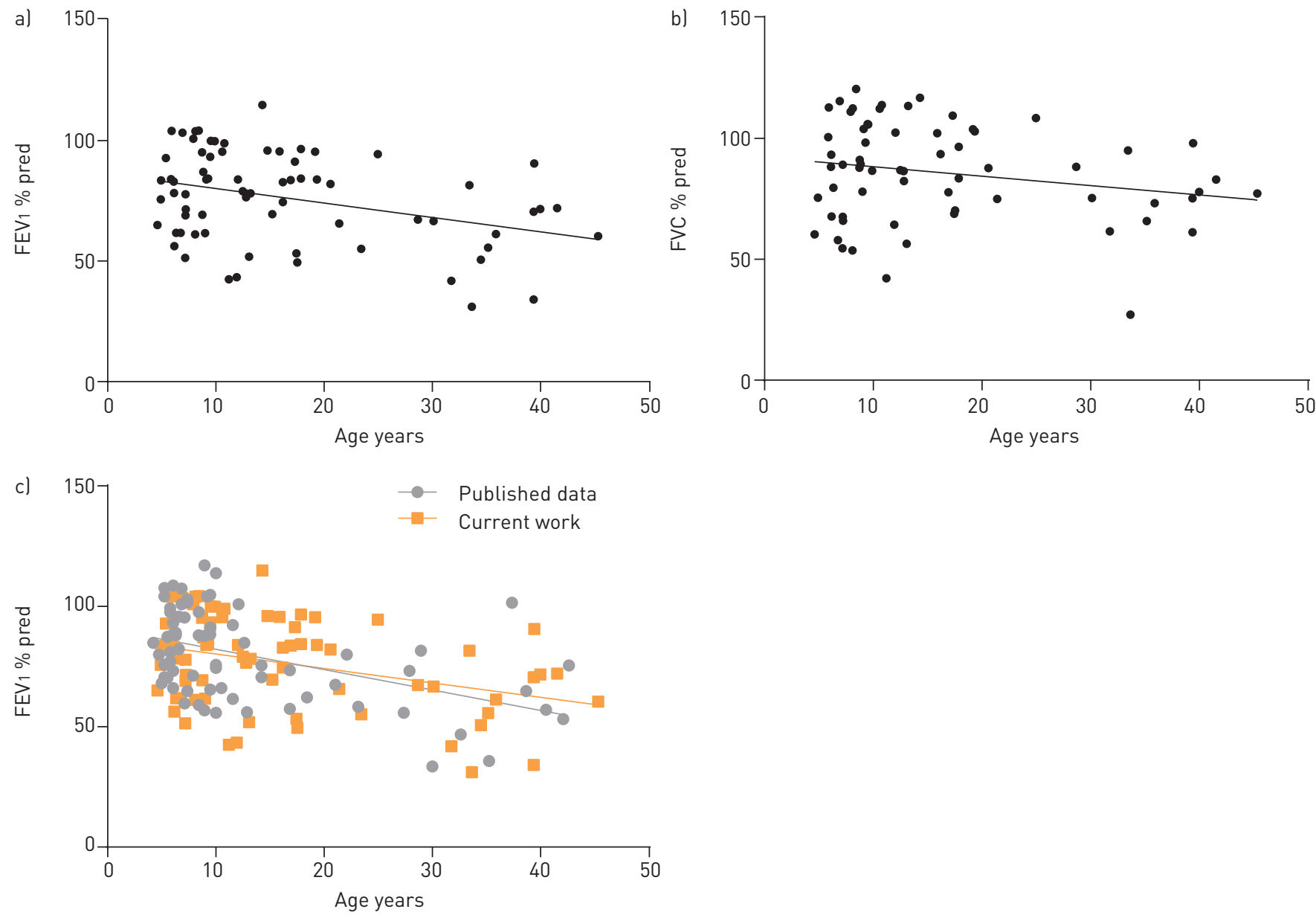

FIGURE 6 Linear regression of lung function parameters versus age. a) Percentage predicted forced expiratory volume in $1 \mathrm{~s}$ (FEV $1 \%$ pred) and b) percentage predicted forced vital capacity (FVC \% pred) were calculated using Global Lung Function Initiative 2012 equations [32]. c) Comparison of $\mathrm{FEV}_{1} \%$ pred values versus age with published data [22] shows a similar decline.

A potential concern for patient self-reported registries is inaccuracy of data. Therefore, we have chosen a clinician-driven data entry system. Confirmation of diagnosis is particularly difficult in PCD because of the high number of genetic and phenotypic subtypes requiring different combinations of methods to establish a diagnosis coupled with limited access to diagnostic services in many countries. In order to reach a high level of diagnostic certainty, we have included all different methods currently used for PCD work-up and established an algorithm to classify cases as confirmed, probable or possible. Furthermore, collaboration of centres within the network will result in a transition of probable or possible to confirmed cases in many instances where limited diagnostic facilities are the main obstacle.

As a limitation, the registry design does not enable patients to directly enter data and therefore a number of patients might not be recruited because they are not cared for by a registry centre. Equally, we cannot approach patients directly who qualify for participation in clinical trials because only strictly anonymised data are stored in the registry database. However, we strongly believe that these limitations are outweighed by the high level of data security that we have achieved in our registry and we ensure patient participation by closely cooperating with patient support groups.

Another reason for a clinician-driven registry is that the data entry system is in the English language. As clinicians and scientists are used to English as the language of medicine, we decided against different language versions. Only QOL-PCD questionnaires will be provided in different languages because these questions will be answered by the patients directly.

Data collection in the registry is not population-based and therefore data might not be representative. However, an international population-based registry for such a rare disorder cannot aim to assemble a high number of individuals internationally. 
Clearing of data entered into the registry is currently under way. We report which methods were used for establishing a PCD diagnosis in figure 4. nNO testing and HVMA were performed most frequently. Both methods exhibited a very low proportion of false-negative test results. $\mathrm{nNO}$ was normal in three individuals with a definite PCD diagnosis, a fact that underlines that PCD cannot be ruled out by demonstration of normal nNO values alone $[25,26]$. HVMA was only normal in individuals who fell into the category of having a possible PCD diagnosis, i.e. some of these four subjects might in fact not be affected by PCD. Of note, we do not have information about the number of individuals 1) with abnormal HVMA findings due to secondary ciliary dyskinesia who were not enrolled into the registry because PCD was excluded by other means or 2) who were not enrolled because of false-normal HVMA findings in phenotypic variants known to be difficult to diagnose by HVMA [2, 3, 7, 11, 12, 27]. Thus, it is not possible to deduce the true sensitivity or specificity of HVMA based on our findings. However, the high proportion of normal TEM findings clearly demonstrates the limited sensitivity of that method in diagnosing the whole spectrum of PCD variants [11, 12, 19, 27, 28]. Identification of ciliary protein mislocalisation by IF requires the use of several antibodies targeting different ciliary components $[2,3,12$, $15,19,29]$. In the registry, IF data were available for a subset of patients. The number of antibodies used in individual patients ranged from two to six. Therefore, the sensitivity of IF might have been higher in subjects with normal IF testing if additional antibodies would have been used for analysis. Bi-allelic disease-causing mutations have been identified in 30 subjects, a number we expect to grow quickly as a result of the increasing use of high-throughput sequencing techniques.

Diagnostic tests recommended for PCD work-up include nNO, HVMA, TEM, IF and genetic analysis [30]. In the majority of subjects, two to three methods were sufficient for a definite PCD diagnosis. 57 individuals had only one or no abnormal test result. These individuals require additional diagnostic efforts to further clarify their disease status, a process now facilitated by the network of different centres collaborating in the registry.

Lung function parameters are key outcome determinants in PCD. Here, we report a decline of FEV $1 \%$ pred with age that is similar to previous reports [22,31]. Pooling our registry data with those published by MARTHIN et al. [22] yielded a mean annual FEV1 \% pred decline of $0.72 \%$ based on a relatively large cohort of 142 PCD patients. FVC \% pred values exhibited a slight annual decline that did not reach statistical significance. Possibly, this is related to the small sample size and will change in the future after incorporation of additional data. Nevertheless, our data support that, in general, PCD lung disease follows more an obstructive than a restrictive pattern.

During the first months of operation, the registry recruited a considerable number of PCD individuals. Of course, we aim to incorporate a much larger number of individuals, not only from BESTCILIA consortium members, but also from other countries. Therefore, we strongly encourage PCD centres worldwide to participate. While the registry is currently run by BESTCILIA members, we are already working on its future structural reorganisation: with an increasing number of participants, it will become impracticable for a single registry administration to monitor all data entered into the registry. Therefore, regional or national subdivisions will be defined that nominate delegates as data administrators and members of the registry steering committee. Naturally, these subdivisions will not be static, i.e. centres from new regions will have the opportunity to designate their own administrators.

In summary, we report the establishment of a comprehensive international registry for PCD as a new promising tool to better understand this rare disorder. Active participation in this registry is highly welcome. For further details and contact information, please refer to the registry homepage www.pcdregistry.eu.

\section{Acknowledgements}

We are grateful to PCD-affected individuals and their families for their participation, and acknowledge continuous support by patient support groups, notably Kartagener Syndrom und Primaere Ciliaere Dyskinesie e.V., North American PCD Foundation, and PCD Family Support Group UK. We thank S. Helms, M. Tekaat and K. Vorspohl for excellent technical assistance.

\section{References}

1 Kuehni CE, Frischer T, Strippoli M-PF, et al. Factors influencing age at diagnosis of primary ciliary dyskinesia in European children. Eur Respir J 2010; 36: 1248-1258.

2 Boon $\mathrm{M}$, Wallmeier J, Ma L, et al. MCIDAS mutations result in a mucociliary clearance disorder with reduced generation of multiple motile cilia. Nat Commun 2014; 5: 4418.

3 Wallmeier J, Al-Mutairi DA, Chen C-T, et al. Mutations in CCNO result in congenital mucociliary clearance disorder with reduced generation of multiple motile cilia. Nat Genet 2014; 46: 646-651.

4 Knowles MR, Daniels LA, Davis SD, et al. Primary ciliary dyskinesia. Recent advances in diagnostics, genetics, and characterization of clinical disease. Am J Respir Crit Care Med 2013; 188: 913-922.

5 Lucas JS, Burgess A, Mitchison HM, et al. Diagnosis and management of primary ciliary dyskinesia. Arch Dis Child 2014; 99: 850-856. 
6 Werner C, Große Onnebrink J, Omran H. Diagnosis and management of primary ciliary dyskinesia. Cilia 2015; 4 $1-9$.

7 Raidt J, Wallmeier J, Hjeij R, et al. Ciliary beat pattern and frequency in genetic variants of primary ciliary dyskinesia. Eur Respir J 2014; 44: 1579-1588.

8 Kott E, Legendre M, Copin B, et al. Loss-of-function mutations in RSPH1 cause primary ciliary dyskinesia with central-complex and radial-spoke defects. Am J Hum Genet 2013; 93: 561-570.

9 Castleman VH, Romio L, Chodhari R, et al. Mutations in radial spoke head protein genes RSPH9 and RSPH4A cause primary ciliary dyskinesia with central-microtubular-pair abnormalities. Am J Hum Genet 2009; 84: 197-209.

10 Olbrich H, Häffner K, Kispert A, et al. Mutations in DNAH5 cause primary ciliary dyskinesia and randomization of left-right asymmetry. Nat Genet 2002; 30: 143-144.

11 Olbrich H, Schmidts M, Werner C, et al. Recessive HYDIN mutations cause primary ciliary dyskinesia without randomization of left-right body asymmetry. Am J Hum Genet 2012; 91: 672-684.

12 Wirschell M, Olbrich H, Werner C, et al. The nexin-dynein regulatory complex subunit DRC1 is essential for motile cilia function in algae and humans. Nat Genet 2013; 45: 262-268.

13 Becker-Heck A, Zohn IE, Okabe N, et al. The coiled-coil domain containing protein CCDC40 is essential for motile cilia function and left-right axis formation. Nat Genet 2011; 43: 79-84.

14 Antony D, Becker-Heck A, Zariwala MA, et al. Mutations in CCDC39 and CCDC40 are the major cause of primary ciliary dyskinesia with axonemal disorganization and absent inner dynein arms. Hum Mutat 2013; 34: 462-472.

15 Merveille A-C, Davis EE, Becker-Heck A, et al. CCDC39 is required for assembly of inner dynein arms and the dynein regulatory complex and for normal ciliary motility in humans and dogs. Nat Genet 2011; 43: 72-78.

16 Hjeij R, Onoufriadis A, Watson CM, et al. CCDC151 mutations cause primary ciliary dyskinesia by disruption of the outer dynein arm docking complex formation. Am J Hum Genet 2014; 95: 257-274.

17 Hjeij R, Lindstrand A, Francis R, et al. ARMC4 mutations cause primary ciliary dyskinesia with randomization of left/right body asymmetry. Am J Hum Genet 2013; 93: 357-367.

18 Omran H, Kobayashi D, Olbrich H, et al. Ktu/PF13 is required for cytoplasmic pre-assembly of axonemal dyneins. Nature 2008; 456: 611-616.

19 Frommer A, Hjeij R, Loges NT, et al. Immunofluorescence analysis and diagnosis of primary ciliary dyskinesia with radial spoke defects. Am J Respir Cell Mol Biol 2015; 53: 563-573.

20 Gliklich RE, Dreyer NA, Leavy MB, eds. Registries for evaluating patient outcomes: a user's guide. 3rd Edn. Rockville, Agency for Healthcare Research and Quality; 2014.

21 Taruscio D, Mollo E, Gainotti S, et al. The EPIRARE proposal of a set of indicators and common data elements for the European platform for rare disease registration. Arch Public Health 2014; 72 : 35.

22 Marthin JK, Petersen N, Skovgaard LT, et al. Lung function in patients with primary ciliary dyskinesia: a cross-sectional and 3-decade longitudinal study. Am J Respir Crit Care Med 2010; 181: 1262-1268.

23 Lucas JS, Behan L, Dunn Galvin A, et al. A quality-of-life measure for adults with primary ciliary dyskinesia: QOL-PCD. Eur Respir J 2015; 46: 375-383.

24 Quittner AL, O’Donnell AE, Salathe MA, et al. Quality of Life Questionnaire-Bronchiectasis: final psychometric analyses and determination of minimal important difference scores. Thorax 2015; 70: 12-20.

25 Marthin JK, Nielsen KG. Choice of nasal nitric oxide technique as first-line test for primary ciliary dyskinesia. Eur Respir J 2011; 37: 559-565.

26 Walker WT, Jackson CL, Lackie PM, et al. Nitric oxide in primary ciliary dyskinesia. Eur Respir J 2012; 40: 1024-1032.

27 Horani A, Brody SL, Ferkol TW, et al. CCDC65 mutation causes primary ciliary dyskinesia with normal ultrastructure and hyperkinetic cilia. PLoS One 2013; 8: e72299.

28 Knowles MR, Leigh MW, Carson JL, et al. Mutations of DNAH11 in patients with primary ciliary dyskinesia with normal ciliary ultrastructure. Thorax 2012; 67: 433-441.

29 Fliegauf M, Omran H. Novel tools to unravel molecular mechanisms in cilia-related disorders. Trends Genet 2006; 22: 241-245.

30 Barbato A, Frischer T, Kuehni CE, et al. Primary ciliary dyskinesia: a consensus statement on diagnostic and treatment approaches in children. Eur Respir J 2009; 34: 1264-1276.

31 Noone PG, Leigh MW, Sannuti A, et al. Primary ciliary dyskinesia: diagnostic and phenotypic features. Am J Respir Crit Care Med 2004; 169: 459-467.

32 Quanjer PH, Stanojevic S, Cole TJ, et al. Multi-ethnic reference values for spirometry for the 3-95-yr age range: the global lung function 2012 equations. Eur Respir J 2012; 40: 1324-1343. 